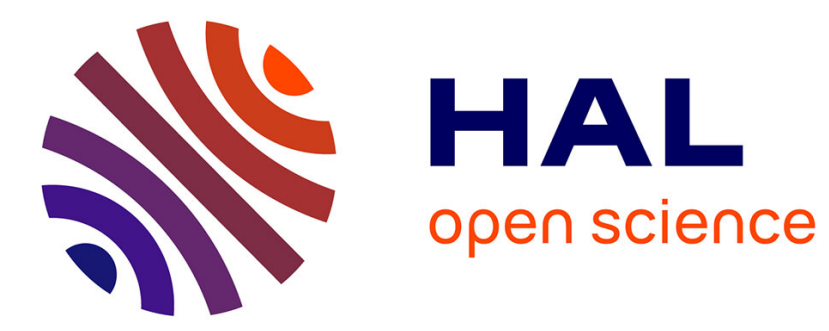

\title{
Generating test cases inside suspicious intervals for floating-point number programs
}

Hélène Collavizza, Claude Michel, Olivier Ponsini, Michel Rueher

\section{To cite this version:}

Hélène Collavizza, Claude Michel, Olivier Ponsini, Michel Rueher. Generating test cases inside suspicious intervals for floating-point number programs. CSTVA, ACM, May 2014, Hyderabad, India. pp.7 - 11, 10.1145/2593735.2593737 . hal-01099503

\section{HAL Id: hal-01099503 https://hal.science/hal-01099503}

Submitted on 28 Jan 2015

HAL is a multi-disciplinary open access archive for the deposit and dissemination of scientific research documents, whether they are published or not. The documents may come from teaching and research institutions in France or abroad, or from public or private research centers.
L'archive ouverte pluridisciplinaire HAL, est destinée au dépôt et à la diffusion de documents scientifiques de niveau recherche, publiés ou non, émanant des établissements d'enseignement et de recherche français ou étrangers, des laboratoires publics ou privés.

\section{(ㅇ)(1) $\$$}

Distributed under a Creative Commons Attribution - NonCommercial - NoDerivatives| 4.0 


\section{Generating Test Cases inside Suspicious Intervals for Floating-Point Number Programs*}

\author{
Hélène Collavizza \\ University of Nice-Sophia \\ Antipolis, I3S/CNRS \\ BP 121, 06903 Sophia \\ Antipolis Cedex, France \\ helen@polytech.unice.fr
}

\author{
Claude Michel \\ University of Nice-Sophia \\ Antipolis, I3S/CNRS \\ BP 121, 06903 Sophia \\ Antipolis Cedex, France \\ Claude.Michel@i3s.unice.fr \\ Michel Rueher \\ University of Nice-Sophia \\ Antipolis, I3S/CNRS \\ BP 121, 06903 Sophia \\ Antipolis Cedex, France \\ Michel.Rueher@i3s.unice.fr
}

\author{
Olivier Ponsini \\ University of Nice-Sophia \\ Antipolis, I3S/CNRS \\ BP 121, 06903 Sophia \\ Antipolis Cedex, France \\ Olivier.Ponsini@i3s.unice.fr
}

\begin{abstract}
Programs with floating-point computations are often derived from mathematical models or designed with the semantics of the real numbers in mind. However, for a given input, the computed path with floating-point numbers may differ from the path corresponding to the same computation with real numbers. State-of-the-art tools compute an overapproximation of the error introduced by floating-point operations with respect to the same sequence of operations in an idealized semantics of real numbers. Thus, totally inappropriate behaviors of a program may be dreaded but the developer does not know whether these behaviors will actually occur, or not. We introduce here a new constraint-based approach that searches for input values hitting the part of the overapproximation where errors due to floating-point arithmetic would lead to inappropriate behaviors. Preliminary results of experiments on small programs with classical floating-point errors are very encouraging.
\end{abstract}

\footnotetext{
* This work was partially supported by ANR VACSIM (ANR11-INSE-0004), ANR AEOLUS (ANR-10-SEGI-0013), and OSEO ISI PAJERO projects.

Part of this work was done while Michel Rueher was visiting professor at NII (National Institute of Informatics).

(c) 2014 Association for Computing Machinery. ACM acknowledges that this contribution was authored or co-authored by an employee, contractor or affiliate of the national government. As such, the Government retains a nonexclusive, royalty-free right to publish or reproduce this article, or to allow others to do so, for Government purposes only.
}

Permission to make digital or hard copies of all or part of this work for personal or classroom use is granted without fee provided that copies are not made or distributed for profit or commercial advantage and that copies bear this notice and the full citation on the first page. To copy otherwise, to republish, to post on servers or to redistribute to lists, requires prior specific permission and/or a fee.

CSTVA '14, May 31, 2014, Hyderabad, India

Copyright 14 ACM 978-1-4503-2847-0/14/05 ...\$15.00.

\section{Categories and Subject Descriptors}

D.2.4 [Software/Program Verification]: Model checking; D.2.5 [Testing and Debugging]: Debugging aids,Testing tools

\section{General Terms}

Verification

\section{Keywords}

Floating-point computations, constraint systems, satisfiable floating-point assignments, unstable programs, test case generation.

\section{INTRODUCTION}

In numerous applications, programs with floating-point computations are derived from mathematical models over the real numbers. However, for some values of the input variables, the result of a sequence of operations over the floating-point numbers can be significantly different from calculations in an idealized semantics of the real numbers. As a consequence, computed paths with floating-point numbers may differ from the paths corresponding to the same computation with real numbers. Identifying these values is a crucial issue for programs controlling critical systems.

Abstract interpretation based error analysis [3, 6, 9] of finite precision implementations compute an over-approximation of the errors due to floating-point operations. Inappropriate behaviors of a program may thus be dreaded but we cannot know whether the predicted unstable behaviors will occur with actual data. This problem is depicted in Fig. 1 where $\left[\underline{x}_{\mathbb{R}}, \bar{x}_{\mathbb{R}}\right]$ stands for the domain of variable $x$ over $\mathbb{R}$, the set of real numbers; and $\left[\underline{x}_{\mathbb{F}}, \bar{x}_{\mathbb{F}}\right]$ stands for the domain of variable $x$ in the over-approximation computed over $\mathbb{F}$, the set of floating-point numbers. In practice, the range of a sequence of operations over the real numbers can often be determined, whether by calculation or from physical limits. A tolerance $\varepsilon$ around this range is usually accepted to take 
into account approximation errors, e.g. measurement, statistical, or even floating-point arithmetic errors. In other words, this tolerance - specified by the user - defines an acceptable loss of accuracy between the value computed over the floating-point numbers and the value calculated over the real numbers. However, values outside the interval $\left[\underline{x}_{\mathbb{R}}-\varepsilon, \bar{x}_{\mathbb{R}}+\varepsilon\right]$ can lead a program to misbehave, e.g. take a wrong branch in the control flow. The values of an approximation over $\mathbb{F}$ that intersect with a forbidden interval are what we call a suspicious interval.

The problem we address in this paper consists in verifying whether a program can actually produce values inside the suspicious intervals $\left[\underline{x}_{\mathbb{F}}, \underline{x}_{\mathbb{R}}-\varepsilon\right]$ and $\left.] \bar{x}_{\mathbb{R}}+\varepsilon, \bar{x}_{\mathbb{F}}\right]$. To handle this problem, we introduce a new constraint-based approach that generates test cases hitting the suspicious intervals in programs with floating-point computations. Our framework reduces this test case generation problem to a constraintsolving problem over the floating-point numbers where the domain of a critical decision variable has been shrunk to a suspicious interval. The generated test cases not only demonstrate that wrong paths can actually be executed but they provide also valuable information for debugging. If no test case can be generated, the suspicious interval can be discarded.

\section{FRAMEWORK FOR TEST CASES GEN- ERATION}

The kernel of our framework is FPCS [14, 13, 1, 12], a constraint solver over floating-point constraints; that's to say a symbolic execution approach for floating-point problems which combines interval propagation with explicit search for satisfiable floating-point assignments. FPCS is used inside the CPBPV [5] bounded model checking framework. CPBPV_FP stands for the adaptation of CPBPV for generating test cases hitting the suspicious intervals in programs with floating-point computations.

The inputs of CPBPV_FP are :

- $P$, an annotated program;

- $x$, a variable used in a critical test; and

- $\left[\underline{x_{F}}, \overline{x_{F}}\right]$, a suspicious interval for $x$.

Annotations of $P$ specify the range of the input variables of $P$ as well as the suspicious interval for $x$. Usually, these annotations are posted just before a critical test using variable $x$.

To compute the suspicious interval for $x$, we approximate the domain of $x$ over the real numbers by $\left[\underline{x}_{\mathbb{R}}, \bar{x}_{\mathbb{R}}\right]$, and over the floating-point numbers by $\left.] \underline{x}_{\mathbb{F}}, \bar{x}_{\mathbb{F}}\right]$. The suspicious intervals for $x$ are $\left.] \underline{x}_{\mathbb{F}}, \underline{x}_{\mathbb{R}}-\varepsilon\right]$ and $\left.] \bar{x}_{\mathbb{R}}+\varepsilon, \bar{x}_{\mathbb{F}}\right]$, where $\varepsilon$ is a tolerance specified by the user.

These approximations are mainly computed with RAICP [15], a hybrid system that combines abstract interpretation and constraint programming techniques in a single static and automatic analysis. $\mathrm{RAICP}$ is substantially more precise than state-of-the-art AI analyzers and CP solvers used separately. Of course, computer algebra systems or interval solvers can also help for computing these approximations. Safe approximations computed with different tools can even be intersected.

CPBPV_FP performs first some pre-processing: $P$ is transformed into DSA-like form. ${ }^{1}$ Loops are handled in CPBPV with standard unfolding and abstraction techniques: if the program contains loops, CPBPV_FP unfolds loops $k$ times. ${ }^{2}$ So, there are no more loops in the program when we start the constraint generation process. Standard slicing operations are also performed to reduce the size of the control flow graph.

In a second step, CPBPV_FP searches for executable paths reaching suspicious interval for $x$. For each of these paths, the collected constraints are sent to FPCS, which solves the corresponding constraint systems over the floating point numbers and returns either a satisfiable instantiation of the input variables of $P$ or an empty set. As said before, FPCS $[14,13,1,12]$ is a constraint solver designed to solve a set of constraints over floating-point numbers without losing any solution. It uses $2 B$-consistency ${ }^{3}$ along with projection functions adapted to floating-point arithmetic $[13,1]$ to filter constraints over the floating-point numbers. FPCS provides also stronger consistencies like $3 B$-consistencies ${ }^{4}$, which allow better filtering results. FPCS allows one to reason correctly over the floating-point numbers with respect to the floatingpoint arithmetic.

CPBPV_FP ends up with one of the following results:

- A test case showing that $P$ can produce a suspicious value for $x$;

- A proof that no test case reaching the suspicious interval can be generated: this is the case if the loops in $P$ cannot be unfolded beyond the bound $k$ (See [5] for details on bounded unfoldings) ;

- An inconclusive answer: no test case could be generated but the loops in $P$ could be unfolded beyond the bound $k$. In other words, the process is incomplete and we cannot conclude whether $P$ may produce a suspicious value.

\section{PRELIMINARY EXPERIMENTS}

We experimented with CPBPV_FP on two cases of floatingpoint arithmetic pitfalls: programs with cancellation and absorption phenomena. ${ }^{5}$

All experiments were done on an Intel Core 2 Duo at 2.8 $\mathrm{GHz}$ with $4 \mathrm{~GB}$ of memory running 64-bit Linux. We assume

\footnotetext{
${ }^{1}$ DSA stands for Dynamic Single Assignment. In DSA-like form, all variables are assigned exactly once in each execution path.

${ }^{2} k$ is usually incremented until a counterexample is found or until the number of time units is large enough for the application.

${ }^{3} 2 B$-consistency [11] states a local property on the bounds of the domains of a variable at a single constraint level. Informally, the domain of variable $x$ is $2 B$-consistent if, for any constraint $c$, there exists at least one value in the domains of all other variables such that $c$ holds when $x$ is set to the upper or lower bound of its domain.

${ }^{4} 3 B$-consistency [11] checks whether $2 B$-Consistency can be enforced when the domain of a variable is reduced to the value of one of its bounds in the whole system.

${ }^{5}$ Absorption in an addition occurs when adding two numbers of very different order of magnitude, and the result is the value of the biggest number, i.e., when $x+y$ with $y \neq 0$ yields $x$. Cancellation occurs in $s-a$ when $s$ is so close to $a$ that the subtraction cancels most of the significant digits of $s$ and $a$.
} 


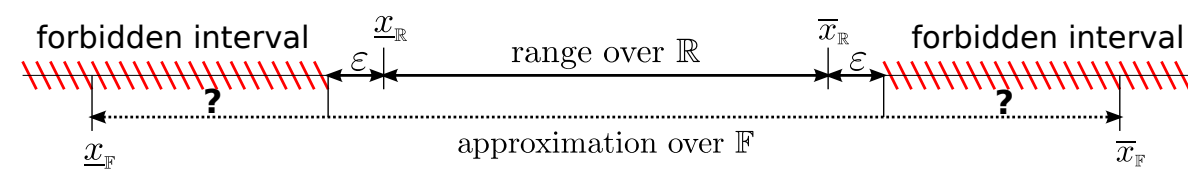

Figure 1: Can the program hit a forbidden zone over the floating-point numbers?

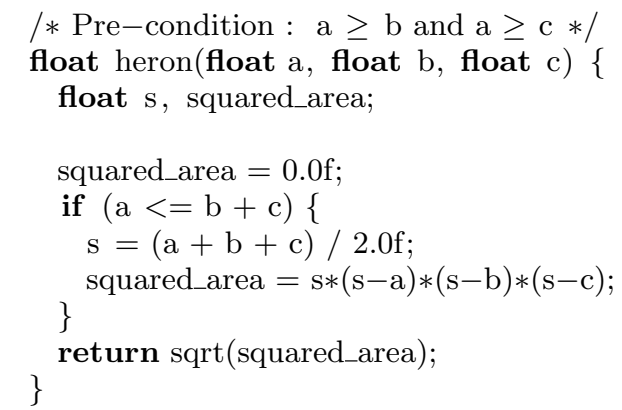

Figure 2: Program Heron

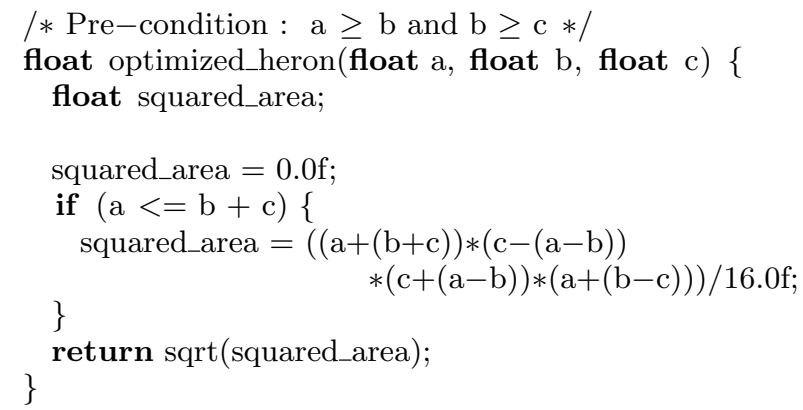

Figure 3: Optimized Heron

C programs handling IEEE 754 floating-point arithmetic, intended to be compiled with GCC without any optimization option and run on an x86_64 architecture managed by a 64-bit Linux operating system. Rounding mode was set to the nearest, i.e., where ties round to the nearest even digit in the required position.

We first describe the programs, and then we compare the performance with existing tools.

\subsection{Program Heron}

The program Heron in Fig. 2 computes the area of a triangle from the lengths of its sides $a, b$, and $c ; a$ being the longest side of the triangle. It use Heron's formula:

$$
\sqrt{s *(s-a) *(s-b) *(s-c)}
$$

where

$$
s=(a+b+c) / 2 .
$$

The test of line 6 ensures that the given lengths form a valid triangle.

Suppose that the input domains are:

- $\mathrm{a} \in[5,10]$;

- b, c $\in[0,5]$.

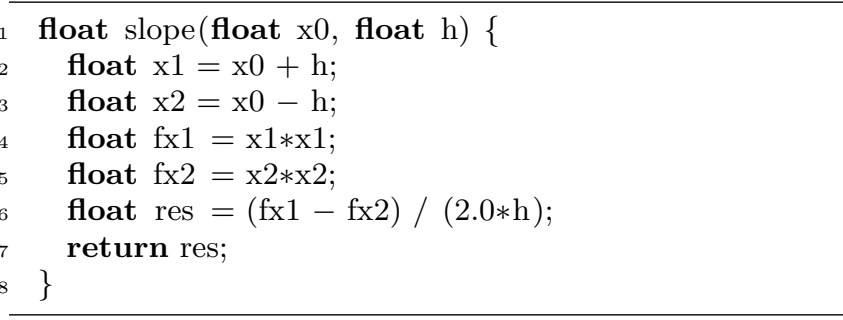

Figure 4: Program slope

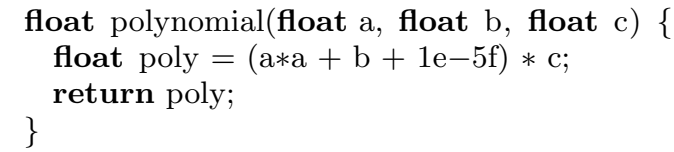

Figure 5: Programs polynomial

Over the real numbers, $\mathbf{s}$ is greater or equal than any of the sides of the triangle and squared_area can neither be negative nor greater than 156.25 since the triangle area is maximized for a right triangle with $b=c=5$ and $a=5 \sqrt{2}$. Value analysis tools FLUCTUAT [6] and RAICP[15] approximate the domain of squared_area to the interval [-1262.21,979.01]. Since this domain is an over-approximation, we do not know whether input values leading to squared_area $<0$ or squared_area $>156.25$ actually exist.

With a tolerance $\varepsilon$ of $10^{-5}$, the suspicious intervals for squared_area are $\left[-1262.21,-10^{-5}\right]$ and $[156.25001,979.01]$. CPBPV_FP managed to generate test cases for both intervals:

- $\mathrm{a}=5.517474, \mathrm{~b}=4.7105823, \mathrm{c}=0.8068917$, and squared_area $=-1.0000001 \cdot 10^{-5}$ for suspicious interval $\left[-1262.21,-10^{-5}\right]$;

- $\mathrm{a}=7.072597, \mathrm{~b}=\mathrm{c}=5$, and squared_area $=156.25003$ for suspicious interval [156.25001, 979.01].

CPBPV_FP could also prove the absence of test cases for a tolerance $\varepsilon=10^{-3}$ with squared_area $>156.25+\varepsilon$.

To limit the loss of accuracy due to cancellation [8], line 8 of this program can be rewritten in the following way : squared_area $=((a+(b+c)) *(c-(a-b)) *(c+(a-b))$

$*(a+(b-v))) / 16.0 f$;

On the optimized version of Heron (Fig. 3), CPBPV_FP still found a test case

- $\mathrm{a}=7.0755463$,

- $\mathrm{b}=4.350216, \mathrm{c}=2.72533$,

- squared_area equals $-1.0000001 \cdot 10^{-5}$. 


\begin{tabular}{|c|c|c|c|c|c|c|}
\hline Name & Condition & FPCS & CDFL & CBMC & CPBPV_FP & sol? \\
\hline \multirow{4}{*}{$\begin{array}{l}\text { slope with } \\
h \in\left[10^{-6}, 10^{-3}\right]\end{array}$} & $r e s<26.0_{f}-1.0_{f}$ & $0.020 \mathrm{~s}$ & $2.014 \mathrm{~s}$ & $1.548 \mathrm{~s}$ & $0.624 \mathrm{~s}$ & yes \\
\hline & $r e s>26.0_{f}+1.0_{f}$ & $0.022 \mathrm{~s}$ & $1.599 \mathrm{~s}$ & $0.653 \mathrm{~s}$ & $0.603 \mathrm{~s}$ & yes \\
\hline & $r e s<26.0_{f}-10.0_{f}$ & $0.007 \mathrm{~s}$ & $0.715 \mathrm{~s}$ & $1.108 \mathrm{~s}$ & $0.588 \mathrm{~s}$ & no \\
\hline & res $>26.0_{f}+10.0_{f}$ & $0.007 \mathrm{~s}$ & $1.025 \mathrm{~s}$ & $1.080 \mathrm{~s}$ & $0.593 \mathrm{~s}$ & no \\
\hline \multirow{4}{*}{$\begin{array}{l}\text { slope with } \\
h \in\left[10^{-9}, 10^{-6}\right]\end{array}$} & res $<26.0_{f}-1.0_{f}$ & $0.010 \mathrm{~s}$ & $0.299 \mathrm{~s}$ & $0.241 \mathrm{~s}$ & $0.593 \mathrm{~s}$ & yes \\
\hline & $r e s>26.0_{f}+1.0_{f}$ & $0.009 \mathrm{~s}$ & $0.333 \mathrm{~s}$ & $0.246 \mathrm{~s}$ & $0.608 \mathrm{~s}$ & yes \\
\hline & $r e s<26.0_{f}-10.0_{f}$ & $0.011 \mathrm{~s}$ & $0.291 \mathrm{~s}$ & $0.224 \mathrm{~s}$ & $0.582 \mathrm{~s}$ & yes \\
\hline & res $>26.0_{f}+10.0_{f}$ & $0.003 \mathrm{~s}$ & $0.342 \mathrm{~s}$ & $0.436 \mathrm{~s}$ & $0.594 \mathrm{~s}$ & yes \\
\hline \multirow[t]{2}{*}{ heron } & squared_aera $<10_{f}^{-5}$ & $0.655 \mathrm{~s}$ & $3.874 \mathrm{~s}$ & $0.280 \mathrm{~s}$ & $1.109 \mathrm{~s}$ & yes \\
\hline & squared_area $>156.25_{f}+10_{f}^{-5}$ & $1.412 \mathrm{~s}$ & $>1200 \mathrm{~s}$ & $34.512 \mathrm{~s}$ & $2.294 \mathrm{~s}$ & yes \\
\hline \multirow[t]{2}{*}{ optimized_heron } & squared_area $<10_{f}^{-5}$ & $0.262 \mathrm{~s}$ & $7.618 \mathrm{~s}$ & $0.932 \mathrm{~s}$ & $0.982 \mathrm{~s}$ & yes \\
\hline & squared_area $>156.25_{f}+10_{f}^{-5}$ & $37.352 \mathrm{~s}$ & $>1200 \mathrm{~s}$ & $>1200 \mathrm{~s}$ & $95.890 \mathrm{~s}$ & no \\
\hline polynomial & $r<1000000000.01_{f}-10_{f}^{-3}$ & $0.006 \mathrm{~s}$ & $0.170 \mathrm{~s}$ & $0.295 \mathrm{~s}$ & $0.605 \mathrm{~s}$ & yes \\
\hline simple_interpolator & $r e s<10_{f}^{-5}$ & $0.017 \mathrm{~s}$ & $0.296 \mathrm{~s}$ & $0.264 \mathrm{~s}$ & $0.613 \mathrm{~s}$ & yes \\
\hline simple_square & $S>1.453125$ & $0.011 \mathrm{~s}$ & -- & $1.079 \mathrm{~s}$ & $0.608 \mathrm{~s}$ & no \\
\hline
\end{tabular}

Table 1: Time required by each solver to solve each problem

when squared_area is set to the interval $\left[-1262.21,-10^{-5}\right]$. CPBPV_FP did also prove that no test case exists with squared_area $\in[156.25001,979.01]$.

\subsection{Program slope}

This program approximates the derivative of a function at a given point $x_{0}$ by computing the slope of a nearby secant line with a finite difference quotient:

$$
f^{\prime}\left(x_{0}\right) \approx \frac{f\left(x_{0}+h\right)-f\left(x_{0}-h\right)}{2 h}
$$

Over the real numbers, the smaller $h$ is, the more accurate the formula is. The program slope in Fig. 4 implements this formula with the square function $f(x)=x^{2}$.

For function $f(x)=x^{2}$ the derivative is given by

$$
f^{\prime}(x)=2 x
$$

which yields exactly 26 for $x=13$. Over the floats, FLuCTUAT and RAICP approximates the return value of the slope program to the interval $[0,25943]$ when $h \in\left[10^{-6}, 10^{-3}\right]$ and $x_{0}=13$.

CPBPV_FP found test cases for the different suspicious intervals with $\epsilon=1$ :

- $\mathrm{h}=7.934571 \cdot 10^{-6}$ and res $=24.999998$ when res is restricted to interval $[0,25]$

- $\mathrm{h}=2.5324175 \cdot 10^{-6}$ and res $=30.126923$ when res is restricted to interval ]27, 25943].

CPBPV_FP proved also that no test case exists with $\epsilon=10$.

The situation gets even worse if we try to increase the accuracy of the formula by decreasing the value of $h$. For an $\epsilon=10$ and $h \in\left[10^{-6}, 10^{-3}\right]$, CPBPV_FP proved that no test case exists whereas for $h \in\left[10^{-9}, 10^{-6}\right]$. CPBPV_FP generated test cases for the different suspicious intervals.

The bad accuracy of program slope comes from a catastrophic cancellation phenomenon: $\mathrm{fx} 1$ and $\mathrm{f} \times 2$ are very close numbers and the subtraction cancels the most significant digits leaving only the digits coming from the rounding of the previous operations.

\subsection{Program polynomial}

The program polynomial (Fig. 5) computes the polynomial

$$
\left(a^{2}+b+10^{-5}\right) * c
$$

and illustrates an absorption phenomenon.

For input domains $a \in\left[10^{3}, 10^{4}\right], b \in[0,1]$ and $c \in$ $\left[10^{3}, 10^{4}\right]$, the minimum value of the polynomial over the real numbers is equal to 1000000000.01 . CPBPV_FP shows that even with a tolerance of $\varepsilon=10^{-3}$, there are input values for which the program computes a result less than $1000000000.01-\varepsilon$.

\subsection{Computation times}

Solving times are given in Table 1.

Programs simple_interpolator and simple_square are two benchmarks extracted from [9]: the first benchmark computes an interpolator, affine by sub-intervals; the second is a rewrite of a square root function used in an industrial context. First column gives the name of the program while the second column gives the condition that is checked. Columns 3 to 6 give the time required to solve the problem by the solver which name the column. Column 7 tells whether the problem has a solution or not. Column FPCS specifies only how much time is spent in the constraint solver. Note that values in column CPBPV_FP include the corresponding time of Column FPCS.

CBMC [4] and CDFL [7] are two state of art software bounded model checkers based on SAT solvers that are able to deal with floating-point computations.

On slope and polynomial, the performance of CBMC and CDFL are very similar to the one of CPBPV_FP: all these systems required little time for finding test cases for these two programs.

Arithmetic expressions are a bit more complex in program heron than in the other benchmarks. CPBPV_FP found nevertheless solutions in reasonable time. But CBMC and CDFL could handle neither the initial nor the optimized version of program heron within a timeout of 20 minutes. 


\section{DISCUSSION}

These preliminary results of experiments are very encouraging: they show that our approach is effective for generating test cases for suspicious values outside the range of acceptable values on on small programs with classical floating-point errors, and thus, to determine whether critical computations are unstable or not.

Performances of FPCS are especially encouraging. The advantage of $\mathrm{CP}$ is that it provides an efficient framework for representing and handling constraints over floating-point numbers. SAT solvers often use bitwise representations of numerical operations, which may be very expensive (e.g., thousands of variables for one equation in CDFL). Another strong point of $\mathrm{CP}$ is its refutation capabilities on constraint systems over the floating point numbers. Of course, experiments on more significant benchmarks and on real applications are still necessary to evaluate the full capabilities and limits of CPBPV_FP.

A new abstract-interpretation based robustness analysis of finite precision implementations has recently been proposed [9] for sound rounding error propagation in a given path in presence of unstable tests. Brain et al [10, 2] have recently introduced a bit-precise decision procedure for the theory of point arithmetic. The core of their approach is a generalisation of the conflict-driven clause-learning algorithm used in modern SAT solver. Their technique is significantly faster than a bit-vector encoding approach. A close connection between our floating-point solvers and the two above mentioned approaches is certainly worth exploring.

\section{REFERENCES}

[1] B. Botella, A. Gotlieb, and C. Michel. Symbolic execution of floating-point computations. Software Testing, Verification and Reliability, 16(2):97-121, 2006.

[2] M. Brain, V. D'Silva, A. Griggio, L. Haller, and D. Kroening. Interpolation-based verification of floating-point programs with abstract cdcl. In Static Analysis - 20th International Symposium SAS, volume 7935 of LNCS. Springer, 2013.

[3] L. Chen, A. Miné, and P. Cousot. A sound floating-point polyhedra abstract domain. In Proceedings of the 6th Asian Symposium on Programming Languages and Systems, APLAS '08, pages 3-18, Berlin, Heidelberg, 2008. Springer-Verlag.

[4] E. Clarke, D. Kroening, and F. Lerda. A tool for checking ANSI-C programs. In TACAS, volume 2988 of LNCS, pages 168-176, 2004.

[5] H. Collavizza, M. Rueher, and P. V. Hentenryck. A constraint-programming framework for bounded program verification. Constraints Journal, 15(2):238-264, 2010.

[6] D. Delmas, E. Goubault, S. Putot, J. Souyris, K. Tekkal, and F. Védrine. Towards an industrial use of FLUCTUAT on safety-critical avionics software. In FMICS, volume 5825 of $L N C S$, pages 53-69. Springer, 2009.

[7] V. D'Silva, L. Haller, D. Kroening, and M. Tautschnig. Numeric bounds analysis with conflict-driven learning. In Proc. TACAS, volume 7214 of Lecture Notes in Computer Science, pages 48-63. Springer, 2012.

[8] D. Goldberg. What every computer scientist should know about floating point arithmetic. ACM Computing
Surveys, 23(1):5-48, 1991.

[9] E. Goubault and S. Putot. Robustness analysis of finite precision implementations. In Programming Languages and Systems - 11th Asian Symposium, APLAS, volume 8301 of $L N C S$, pages 50-57. Springer, 2013.

[10] L. Haller, A. Griggio, M. Brain, and D. Kroening. Deciding floating-point logic with systematic abstraction. In Formal Methods in Computer-Aided Design, FMCAD, pages 131-140. IEEE, 2012.

[11] O. Lhomme. Consistency techniques for numeric CSPs. In 13th International Joint Conference on Artificial Intelligence, pages 232-238, 1993.

[12] B. Marre and C. Michel. Improving the floating point addition and subtraction constraints. In $C P$, volume 6308 of LNCS, pages 360-367. Springer, 2010.

[13] C. Michel. Exact projection functions for floating-point number constraints. In 7th International Symposium on Artificial Intelligence and Mathematics, 2002.

[14] C. Michel, M. Rueher, and Y. Lebbah. Solving constraints over floating-point numbers. In $C P$, volume 2239 of LNCS, pages 524-538. Springer Verlag, 2001.

[15] O. Ponsini, C. Michel, and M. Rueher. Refining abstract interpretation based value analysis with constraint programming techniques. In M. Milano, editor, 18th International Conference on Principles and Practice of Constraint Programming (CP 2012), volume 7514 of Lecture Notes in Computer Science, pages 593-607. Springer, 2012. 